\title{
OTIMIZAÇÃO DA PRODUÇÃO ACADÊMICO-CIENTÍFICA PARA MECANISMOS DE BUSCA ACADÊMICOS (ASEO)*
}

\author{
EMANUELLE TORINO** \\ GUSTAVO LUNARDELLI TREVISAN ${ }^{* * *}$ \\ SILVANA APARECIDA BORSETTI GREGORIO VIDOTTI ${ }^{* * * *}$
}

\begin{abstract}
RESUMO
A crescente disponibilização de websites e conteúdos informacionais na web torna necessária a adoção de estratégias e técnicas para que os mecanismos de busca possam rastrear e coletar informações contidas, por exemplo, no código fonte, na URL e nos links das páginas e para que tenham melhor ranqueamento na página de resultados. Tais estratégias e técnicas podem ser adotadas no contexto da otimização de conteúdos para mecanismo de busca, Search Engine Optimization. De igual maneira, há conteúdos oriundos da produção acadêmico-científica disponibilizados em ambientes informacionais digitais web, que podem ser recuperados por mecanismos de busca acadêmicos e, cujo ranqueamento na página de resultados pode ocorrer com a utilização de estratégias e técnicas de Academic Search Engine Optimization. Assim, na perspectiva ampliação da visibilidade e do uso da produção acadêmico-científica este estudo objetiva apresentar estratégias e técnicas de otimização de conteúdos para mecanismos de busca acadêmicos, sob a ótica do autor. Para tanto, utiliza-se da pesquisa bibliográfica para discorrer acerca da temática e apresentar as discussões, com base na Ciência da Informação. Como resultados, temse um modelo conceitual de otimização da produção acadêmico-científica para mecanismos de busca acadêmicos, sob a perspectiva do autor, no qual são apresentadas as estratégias e técnicas de otimização de conteúdos para mecanismo de busca acadêmico que podem ser utilizados para a ampliação da visibilidade, do uso e da possibilidade de citação.
\end{abstract}

PALAVRAS-CHAVE: Otimização para mecanismo de busca acadêmico. Otimização da produção acadêmico-científica. Preparação de conteúdos acadêmico-científicos. Disseminação da produção acadêmico-científica.

\footnotetext{
* Este trabalho foi originalmente publicado e apresentado VIII Seminário em Ciência da Informação (SECIN) da Universidade Estadual de Londrina (UEL), em agosto de 2019.

* Universidade Tecnológica Federal do Paraná (UTFPR); Universidade Estadual Paulista - Unesp

*nt* Universidade Estadual Paulista - Unesp

${ }^{* * * *}$ Universidade Estadual Paulista - Unesp
} 


\title{
OPTIMIZATION OF ACADEMIC-SCIENTIFIC PRODUCTION FOR ACADEMIC SEARCH ENGINES (ASEO)
}

\begin{abstract}
The growing availability of websites and informational websites makes it necessary to adopt new strategies and techniques so that the searching mechanisms can track and collect information contained in the URL source code, and in the page links. In order to have a better outcome in the results page, these strategies and techniques may be adopted, relating to the optimization of searching contents, Search Engine Optimization. Likewise, there are some contents originated from the scientific-academic production available in digital web informational sites, that can be recovered by academic search mechanism and which rank in the page results can happen with the use of the strategies and techniques of the Academic Search Engine Optimization. Therefore in the sight of the visibility broaden and the use of the scientific-academic production this study aims to present the optimization of strategies and techniques for academic searching content, by the author's point of view. For this purpose, the bibliographic research is used to talk about the subject and to present conversations related to the Information Science. As results, there is a conception of an optimized model of search engine for scientific-academic production, in which, by the author's point of view, strategies and techniques of optimization of contents for academic search mechanism is presented in a way that it broaden the visibility and raises the possibility of being quoted.
\end{abstract}

KEYWORDS: Search engine optimization. Optimization for academic search engine. Optimization of academic-scientific production. Preparation of academic-scientific content. Dissemination of academic-scientific production.

\section{INTRODUÇÃO}

Na sociedade contemporânea, os mecanismos de busca (MB) propiciam a recuperação e o acesso à informação em ambientes informacionais digitais que constituem ecologias informacionais complexas, definidas por Oliveira e Vidotti (2016, p. 91) como "[...] um conjunto de espaços, ambientes, canais, mídias, tecnologias e sujeitos com seus comportamentos, todos interligados e conectados de maneira holística pela informação."

No contexto dos ambientes informacionais da web, os mecanismos de busca utilizam crawlers para indexar os conteúdos dos websites, por meio de algoritmos de extração de dados e informações contidas, por exemplo, no código fonte, na URL e nos links das páginas.

Existem mecanismos de busca acadêmicos (MBA) que indexam, exclusivamente, conteúdos acadêmicos e científicos, tais como artigos, livros, teses, dissertações e materiais educacionais. 
Tais recursos são oriundos de bibliotecas e repositórios digitais, revistas científicas eletrônicas, bases indexadoras, websites acadêmicos, dentre outros ambientes acadêmicos e científicos. Como exemplos de MBA temos Google Scholar ${ }^{1}$, CiteSeer ${ }^{2}$, Microsoft Academic Search ${ }^{3}$, Base ${ }^{4}$ e RefSeek ${ }^{5}$.

A partir de uma requisição realizada por um usuário, utilizando uma expressão de busca, por meio da interface de mecanismos de busca - gerais ou acadêmicos - o processo de recuperação da informação é ativado para exibir conteúdos indexados pelo MB ou MBA. Para tanto, cada mecanismo de busca, sejam eles gerais ou acadêmicos, utiliza algoritmos que atendem a critérios específicos para coleta, recuperação e ranqueamento dos resultados na Search Engine Results Page (SERP).

Com intuito de melhorar o ranqueamento das páginas web na SERP, são utilizadas estratégias e técnicas de otimização para mecanismos de busca, Search Engine Optimization (SEO), e, em específico, estratégias e técnicas de otimização para mecanismos de busca acadêmicos, Academic Search Engine Optimization (ASEO).

Assim, nas perspectivas de ampliação da visibilidade e de uso da produção acadêmico-científica, este estudo objetiva apresentar estratégias e técnicas de otimização de conteúdos para mecanismos de busca acadêmicos (ASEO), sob a ótica do autor.

O estudo utilizou-se de revisão de literatura nas áreas de Ciência da Informação, Ciência da Computação e Marketing Digital para embasar os conceitos apresentados e propor técnicas de ASEO para os autores de trabalhos acadêmico-científicos.

Os resultados apresentam técnicas e estratégias de ASEO pré e pós-publicação que podem ser adotadas pelos autores visando à otimização de suas produções acadêmico-científicas para mecanismos de busca acadêmicos.

\section{MECANISMOS DE BUSCA ACADÊMICOS}

O mecanismo de busca, embora em sua interface possa ser semelhante a um website, constitui-se em software utilizado para percorrer a web utilizando crawlers e indexar conteúdos,

\footnotetext{
${ }^{1}$ Disponível em: http://scholar.google.com/. Acesso em: 28 maio 2019.

${ }^{2}$ Disponível em: http://citeseerx.ist.psu.edu/index. Acesso em: 28 maio 2019.

${ }^{3}$ Disponível em: https://academic.microsoft.com/. Acesso em: 28 maio 2019.

${ }^{4}$ Disponível em: https://www. base-search.net/. Acesso em: 28 maio 2019.

${ }^{5}$ Disponível em: https://www.refseek.com/. Acesso em: 28 maio 2019.
} 
armazenando-os em uma base de dados que é acionada para apresentar os resultados na SERP sempre que uma consulta é executada.

Ledford (2009, p. 5, tradução nossa) apresenta o back-end de um MB como "[...] um software que usa algoritmos para localizar e coletar informações sobre páginas da web. [...] Essas informações são indexadas e armazenadas em um banco de dados."

Neste contexto, as informações coletadas são indicadores dos conteúdos disponíveis, como título, palavras-chave, URL e links, que os representam na estrutura do banco de dados e são acionados quando uma consulta (query) é realizada.

Esta consulta é realizada pela interface, chamada por Ledford (2009) de front-end, na qual o usuário insere a estratégia de busca e a partir dela "[...] o algoritmo examina as informações armazenadas no banco de dados do back-end e recupera links para páginas da web que correspondem ao termo de pesquisa que o usuário inseriu". (p. 5, tradução nossa). Os resultados da consulta são apresentados na SERP, que exibe "[...] a coleção das páginas que são retornadas com os resultados da pesquisa depois que um usuário insere um termo ou expressão de busca e clica no botão de pesquisa". (LEDFORD, 2009, p. 5, tradução nossa).

O desenvolvimento das tecnologias e da web ocasionou também o desenvolvimento dos MB. Monteiro et al. (2011, p. 2548) apresentam-nos em três gerações:

$\mathrm{Na}$ primeira, havia principalmente uma página de dados (texto e formatação) e esses mecanismos estavam próximos ao modelo clássico de recuperação da informação, no qual não considera aspectos inerentes ao contexto de busca, ou seja, a Interação Humano-Computador e os aspectos cognitivos, ou os aspectos semânticos da busca. $\mathrm{Na}$ segunda geração, os mecanismos passaram a suportar buscas navegacionais e informacionais. $\mathrm{Na}$ atual terceira geração [...] há tentativas de apresentação de dados de diversas fontes, com o objetivo de contextualizar o indivíduo no que diz respeito a sua busca e, ainda, compreender a necessidade por trás da busca, por meio de análise semântica, determinação do contexto entre outros aspectos.

Essa evolução permite compreender a relevância da otimização de conteúdos para $\mathrm{MB}$, uma vez que os algoritmos se aprimoram continuamente no sentido de apresentar na SERP os resultados que melhor respondam à estratégia de busca do usuário. Contudo, para que isso ocorra, a estruturação das páginas web, dos websites e, no 
contexto deste estudo, da produção acadêmico-científica precisa ser realizada de forma a representar adequadamente o conteúdo e seu contexto, trazendo significado para a interação do usuário com a informação recuperada, o que se aplica a MBs e MBAs.

Recuperação da Informação tem suas origens na Ciência da Computação (CC), embora haja diversos autores que abordam a temática na perspectiva da Cl. Para Mooers (1951, p. 25, tradução nossa),

A recuperação da informação abrange os aspectos intelectuais da descrição da informação e sua especificação para busca, e também quaisquer sistemas, técnicas ou máquinas que são empregados para realizar a operação.

Na perspectiva do usuário, a recuperação da informação tem início na elaboração de uma expressão de busca e culmina no recebimento dos resultados encontrados pelo sistema de recuperação da informação e na sua apresentação na SERP.

A partir da década de 1960, a Ciência da Informação começou a abarcar questões pertinentes à organização de sistemas de informação, possibilitando novos olhares para o desenho da recuperação da informação, como parte crucial para a documentação e organização do conhecimento em diferentes ambientes informacionais. (MONTEIRO et al., 2017).

Na perspectiva da Ciência da Informação, a RI é investigada por meio de uma interpretação voltada para o usuário e sua compreensão da busca e utilização da informação. No entendimento do usuário como ponto central no processo da RI, Ingwersen (1992) propõe 0 entendimento do conceito, fundamentado no processamento daquilo que é executado por um sistema, simulando o processamento mental do homem para entender o mundo.

\section{OTIMIZAÇÃO DE CONTEÚDOS PARA MECANISMOS DE BUSCA}

Otimização de conteúdos para mecanismos de busca, do inglês Search Engine Optimization (SEO), consiste na aplicação de estratégias e técnicas para que websites sejam mais bem ranqueados pelos mecanismos de busca.

Após disponibilizados na web, os websites são indexados por mecanismos de busca e podem ser recuperados a partir de uma consulta (query). Neste sentido, ao discorrer sobre SEO, Ledford (2009, p. 40) afirma que: 
Alguns especialistas em SEO acreditam que tudo se resume a otimizar o conteúdo do seu site para chamar a atenção dos rastreadores e indexadores que indexam sites. Outros acham que é o número de links de qualidade que você pode gerar em seu site. $O$ SEO orgânico é, na verdade, uma combinação desses e de outros elementos, como a marcação de sites, que colocará naturalmente o seu site nos rankings dos mecanismos de busca. Quão alto nesses rankings depende de quão bem você projeta seu site.

Isso posto, clarifica-se que, independente de qualquer ação, as páginas disponíveis na web serão, em algum momento, indexadas por mecanismos de busca, contudo não há qualquer indicação do grau de relevância de um website para um MB; para otimizar esta ação utilizam-se estratégias e técnicas SEO, para que o website seja mais bem posicionado na SERP.

Para melhor entendimento, o SEO está estruturado em fatores internos e externos: (LEDFORD, 2009; JERKOVIC, 2010; ENGE; SPENCER; STRICCHIOLA, 2015).

a) fatores internos ou on-page: são elementos relacionados a aspectos internos do website e que podem ser controlados pelo desenvolvedor, dentre os quais se destacam:

- adoção de Uniform Resource Locator (URL) amigável, preferencialmente textual e que contenha o assunto principal do website;

- definição do assunto/tema abordado no website, que deve constar na tag título, do head, contido no código fonte;

- estrutura e hierarquia das páginas devidamente organizadas no código fonte, utilizando, por exemplo, header h1, h2, h3. Os títulos devem ser claros, precisos e conter até 60 caracteres, a quantidade máxima exibida no $\mathrm{MB}$;

- cada página deve conter uma meta descrição, ou seja, um resumo com até 160 caracteres, que será exibido na SERP como elemento motivador de acesso à página pelo usuário;

- palavras-chave derivadas do tema, que devem ser utilizadas no conteúdo com frequência a fim de determinar a relevância, sem se tornarem demasiadamente repetitivas. Tais palavras-chave devem ser definidas com foco no usuário do website, para tanto devem ser realizados monitoramentos do próprio website e de concorrentes, utilizando ferramentas específicas;

- no conteúdo considerar: qualidade, autenticidade, 
legibilidade, atualidade, extensão, campo semântico, uso adequado de imagens e suas descrições textuais (ALT, título alternativo, nome do arquivo e legenda). Como parte do conteúdo, podem ser utilizados links para o próprio website (interlink) ou para páginas externas (link building), para tanto, utilizam-se textos âncora, visíveis e clicáveis que uma vez acionados direcionam o usuário para as páginas especificadas;

- oferecer os arquivos sitemap indicando as páginas contidas no website e robots.txt para indicar quais páginas não devem ser indexadas pelo $\mathrm{MB}$;

b) fatores externos ou off-page, também chamados link building e backlink: são aspectos externos e de entrada ao website, realizados por outros sites, e por isso fogem do controle do desenvolvedor, embora tenham relação com o conteúdo. Os fatores off-page não consistem em simples links, e sim no reconhecimento da autoridade do website em relação ao assunto disponível, por isso os MB também verificam as páginas que o indicam para identificar se atendem a alguns critérios, dentre os quais idoneidade e ranqueamento, para assim considerá-los na análise do website. O link building pode ser feito no conteúdo ou em comentário em páginas externas, sejam elas outros domínios, redes sociais ou blogs. Para tanto, é necessário:

- possuir conteúdo relevante, de forma a atrair usuários e mantê-lo no website;

- investir em relacionamento e estratégias de divulgação, como participar de blogs e fóruns da área do website, de forma a atrair tráfego para o website;

- escrever conteúdos para outras páginas relacionadas ao assunto/tema do website como forma de gerar links para a página;

- compartilhar os conteúdos em redes sociais utilizando links para o website;

- disponibilizar botões de compartilhamento para que os usuários também possam criar links para o website $e$ aumentar o tráfego para ele.

É sabido, ainda, que existem atitudes tomadas pelo desenvolvedor consideradas violações ou black hat, que consistem técnicas antiéticas aplicadas ao código fonte, não visíveis a humanos, realizadas visando promover o ranqueamento do website 
no SERP, sem otimizar o conteúdo com fatores on ou off-page. Embora possa em princípio parecer ser uma boa estratégia, a prática é condenável e pode gerar sanções como a exclusão definitiva do MB.

Vale destacar que, no contexto do SEO, há a menção à experiência do usuário, tratada, contudo, em uma perspectiva de eficiência do website, considerando aspectos de marketing digital, tais como: tempo de resposta do site, uso de pop-up, responsividade, facilidade de leitura rápida, presença massiva de links, design amigável, e uso adequado de cores.

Isso se justifica pela origem do SEO, do marketing digital, cuja finalidade é alavancar negócios, como a venda de produtos ou serviços utilizando-se de websites, e para tanto utiliza estratégias técnicas para otimizar o posicionamento das páginas na SERP.

Uma das características básicas do SEO é a chamada taxa de conversão, ou seja, conseguir que os usuários do ambiente realizem a ação desejada quando da disponibilização do conteúdo. Assim, embora o SEO trabalhe com técnicas de otimização de conteúdos, sua realização justifica-se pela necessidade de que o usuário localize o website a partir do seu posicionamento na SERP e interaja com ele.

Destaca-se que os websites possuem conteúdos dinâmicos e passíveis de alteração contínua, de forma que as estratégias e técnicas de SEO podem ser aplicadas, o website monitorado e novas decisões tomadas para influenciar o ranqueamento no MB. Ledford (2009) esclarece que, após aplicar as técnicas SEO, é importante verificar se os objetivos foram atingidos e o posicionamento na SERP melhorado, para tanto sugere que a avaliação seja realizada trimestralmente.

Considerando a crescente disponibilidade de conteúdos acadêmico-científicos na web e mecanismos de busca específicos para este fim, as técnicas de SEO foram adaptadas, dando lugar ao Academic Search Engine Optimization (ASEO), objetivando otimizar a visibilidade das publicações científicas, sobretudo artigos, dentre outras formas de produção neste campo, como dissertações e teses. (BEEL; GIPP; WILDE, 2010; CODINA, 2017). É válido observar que, embora existam diversos mecanismos de busca acadêmicos na web, o Google Scholar é o MBA mais utilizado para estudos acerca do ASEO. (BEEL, GIPP, WILDE, 2010; CODINA, 2017).

Para Beel, Gipp e Wilde (2010, p. 176, tradução nossa), "Academic search engine optimization (ASEO) é a criação, publicação e modificação da literatura acadêmica de uma maneira 
que facilite aos mecanismos de busca acadêmicos a coleta e a indexação." Os autores apontam quatro aspectos significativos acerca da ASEO, que os diferenciam do SEO:

a) ausência de um MBA líder ocidental: esta ausência torna a otimização dos conteúdos mais complexa, visto que pode não existir um parâmetro entre os MBAs, o que tornaria diferente o processo de ranqueamento na SERP;

b) indexação em bases de dados invisíveis: os objetos resultantes da atividade acadêmico-científica são publicados em fontes sobre as quais o autor não exerce controle, sejam elas periódicos, repositórios digitais, e apenas os detentores de tais ambientes podem tomar medidas para que eles sejam indexados nos MBAs. Neste sentido, compete ao autor planejar na etapa pré-publicação alternativas para que seus objetos sejam visíveis na web;

c) conteúdos publicados são estáticos: no contexto acadêmico-científico o autor possui autonomia acerca do conteúdo até a etapa de publicação, posteriormente, independente do modelo de negócio estabelecido com o editor, não há qualquer possibilidade de alteração do texto publicado, o que exige atenção nas estratégias adotadas antes da publicação e que afetarão a otimização para os MBAs;

d) indexação parcial de conteúdo: nos MBAs há a garantia de que os metadados preenchidos na submissão para a publicação, sobretudo título e resumo, serão utilizados para a indexação. Isso exige do autor maior atenção na definição desses campos, contudo não se pode esquecer que o conteúdo é fundamental em todo o contexto.

Para Codina (2017, tradução nossa, grifos do autor), ASEO é "[...] o conjunto de práticas que promovem a visibilidade e a facilidade de acesso das produções acadêmicas, tipicamente, artigos de revistas científicas, mas também monografias, relatórios e qualquer outra classe de produto resultante de uma investigação." Para o autor, os principais objetivos do ASEO são o aumento da visibilidade da produção acadêmica e a probabilidade de que esta produção seja citada, o que consiste em um ciclo.

Para tanto, segundo Codina (2017), o ASEO utiliza fatores internos e externos e, de modo adicional e exclusivo, há o acréscimo da identidade digital, que consiste em adotar uma forma de citação 
pela qual o autor será reconhecido, preferencialmente composta por dois nomes, e criar um perfil utilizando um identificador persistente no qual possam ser indicadas as formas variantes do nome do autor, com a finalidade de desambiguar a autoria.

Ademais, destaca-se que, assim como o SEO, o ASEO tem em conta a taxa de conversão, contudo a ação desejada é que os usuários do ambiente utilizem e citem os conteúdos disponibilizados. Para tanto, deve-se atentar para o fato de que, diferente dos websites, as produções acadêmico-científicas são estáticas e não podem ser alteradas após a publicação, o que aumenta a relevância das estratégias e técnicas pré-publicação, cuja influência será direta no ranqueamento no MBA.

Ainda, o uso e a práxis do ASEO têm um viés voltado à questão da ampliação da visibilidade científica e algumas práticas já são realizadas por pesquisadores, sobretudo na forma de disseminação da produção científica pós-publicação, utilizando-se de páginas pessoais, blogs ou mesmo redes sociais para disponibilizar o link de acesso ao trabalho, o que consiste na estratégia de link building e, ainda, a disponibilização cada vez mais frequente dos resultados de pesquisa em repositórios digitais. No que se refere à identidade digital, o Open Research and Contributors Identification (ORCID iD) tem sido amplamente adotado, dada a recomendação por instituições públicas, dentre elas a Coordenação de Aperfeiçoamento de Pessoal de Nível Superior (CAPES).

Discutiremos, a seguir, as estratégias e técnicas de ASEO que podem ser aplicadas ao contexto acadêmico-científico visando melhorar o posicionamento dos objetos produzidos em benefício da visibilidade e da citação dos autores.

\section{RESULTADOS: APRESENTAÇÃO E DISCUSSÃO}

Considerando a ausência de um mecanismo de busca acadêmico que tenha liderança mundial (BELL; GIPP; WILDE, 2010), o presente estudo não utilizou um MBA específico para a aplicação, enfocando-se nas práticas de ASEO a serem adotadas pelo autor para favorecer a otimização da sua produção acadêmicocientífica para MBA.

A Figura 1 apresenta um modelo conceitual de otimização da produção acadêmico-científica para MBA sob a perspectiva do autor, considerando duas etapas, pré-publicação e pós-publicação. 
Figura 1 - Otimização da Produção Acadêmico-Científica para MBA

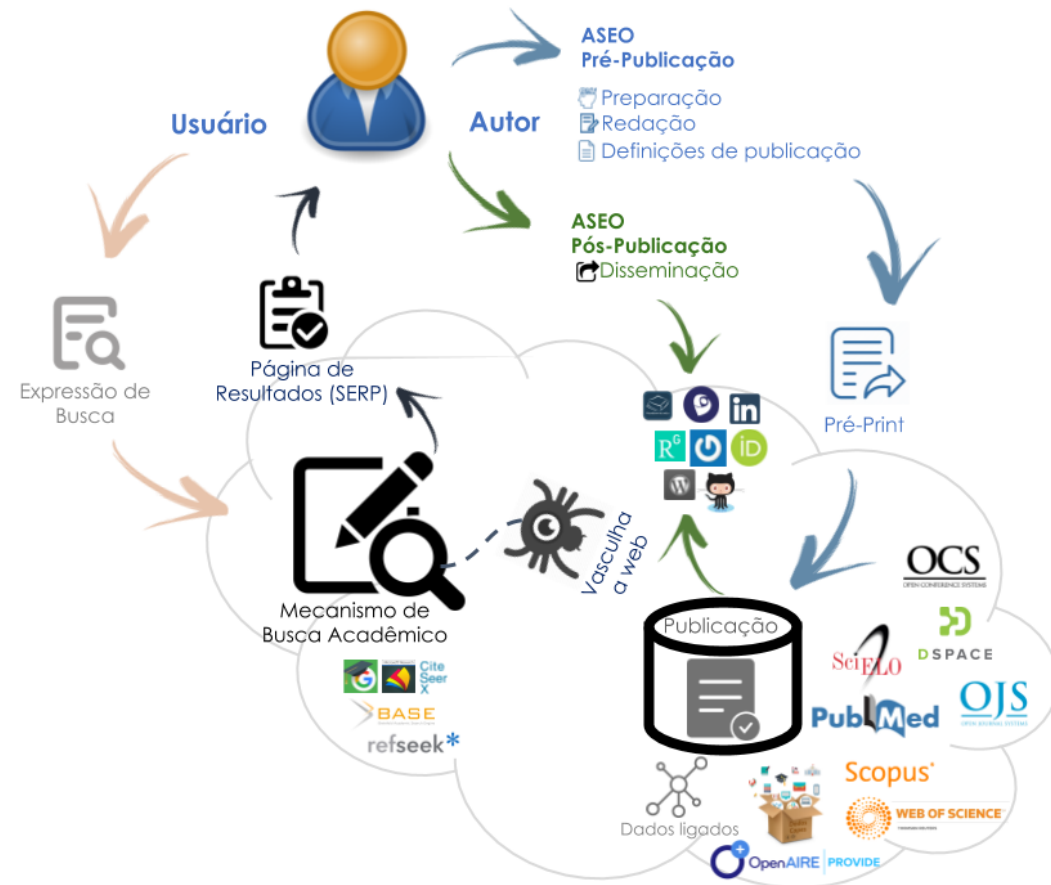

Fonte: Autoria própria (2019).

Codina (2017) estabelece um paralelo entre a etapa pré-publicação e os fatores on-page do SEO, uma vez que, nesta etapa, as decisões tomadas pelo autor podem influenciar diretamente no ranqueamento do objeto na SERP.

Não é objetivo deste estudo discutir como o autor deve conduzir as fases de concepção de sua pesquisa ou a redação do objeto a ser publicado. Desta forma, apresentamos estratégias e técnicas que podem ser adotadas, com base no ASEO, para possibilitar que as produções tenham maior possibilidade de indexação e ranqueamento nos MBAs. (BELL; GIPP; WILDE, 2010; CODINA, 2017). Vale destacar que, uma vez adequadamente ranqueado na SERP, o objeto publicado estará visível aos usuários e poderá ser por eles utilizado, gerando a possibilidade de citação, o que consiste, no contexto do ASEO, na taxa de convergência.

A etapa pré-publicação é totalmente autônoma e as estratégias e técnicas podem ser aplicadas pelo autor, pois cabe a ele todas 
as decisões acerca do conteúdo que está sendo otimizado. Esta etapa é iniciada na preparação do objeto, e a partir da definição da temática a ser abordada devem ser definidas palavras-chave relevantes e que serão utilizadas. Esta definição levará em conta o público-alvo do conteúdo a ser otimizado, bem como a forma com que ele é pesquisado pelos usuários. Para a definição, podem ser utilizadas outras publicações que abordem a mesma temática, bases de dados, vocabulários controlados ou a própria web.

Durante a redação do pré-print, as palavras-chave selecionadas devem ser utilizadas obrigatoriamente nos campos título, resumo e palavras-chave, bem como ao longo do texto, o que reforça a sua relevância. $O$ uso de sinônimos para as palavras-chave pode ser realizado no resumo e no conteúdo, mas em quantidade reduzida.

O nome do autor deve ser indicado de maneira consistente e, para tanto, recomenda-se o uso de identificador persistente para que não haja ambiguidade e para possibilitar que os MBAs gerem adequadamente as métricas de citação.

Codina (2017) destaca que a visibilidade de conteúdos científicos se justifica pela possibilidade de citação e que erros nas citações geradas pelas formas variantes de nomes compostos ocasionam prejuízos ao pesquisador. Neste sentido, recomenda o uso do ORCID iD como identificador persistente de autor, para desambiguidade de nome e indicação de formas variantes, bem como a adoção do perfil no Google Scholar para que as produções sejam reunidas indicando as métricas de citação.

De igual maneira, deve-se utilizar adequadamente os nomes dos autores citados no texto e, quando possível, indicar, nas referências, o link para o texto completo do trabalho citado na fonte original de publicação, de forma a gerar link build e aumentar o tráfego para a página. Caso o autor do trabalho utilize outros trabalhos por ele publicados, estes devem ser adequadamente citados e referenciados.

As tabelas e ilustrações devem ser legíveis por máquinas, para que também possam ser indexadas por MBAs. Além disso, tal prática privilegia o uso do conteúdo por usuários que necessitam de ferramentas de acessibilidade, como leitores de texto.

Na formatação, utilizar a estrutura comum para trabalhos da área para que os mecanismos de busca e os próprios leitores estejam familiarizados com a forma de apresentação do conteúdo.

Após finalizada a redação, devem ser realizadas as definições de publicação, como parte do processo de otimização 
do conteúdo para MBA. Cabe ao autor definir qual será a fonte de publicação para o seu conteúdo, tendo em conta características como: modelo de negócio, formas de tratamento dos direitos autorais, disponibilidade em acesso aberto, indexação por MBA, tempo entre recebimento e publicação, e outras que sejam convenientes.

Codina (2017) recomenda a adoção de publicações em acesso aberto, quer seja em periódicos (via dourada) ou repositórios digitais (via verde), sempre que couber ao autor esta escolha. Neste sentido, como já é vivenciado internacionalmente, as agências de fomento brasileiras começam a requerer a disponibilização de versões de trabalhos em repositórios digitais como condição para obtenção de recursos.

Destaca-se que, após a publicação, não caberá ao autor qualquer forma de alteração ao conteúdo publicado, o que reforça a importância das definições tomadas no momento de escolha da fonte de publicação.

Definida a fonte de publicação, caberá ao autor a submissão do pré-print, realizada mediante o preenchimento dos metadados básicos utilizados para a indexação pelos MBAs: título, resumo e palavras-chave, cujas estratégias de otimização foram abordadas anteriormente.

Neste sentido, Torino e Vidotti (2019) discutem o papel do autor na representação da informação em ambientes de publicação científica, cujas técnicas são oriundas da área de Ciência da Informação, apontam que o tratamento inadequado realizado por ele afeta a interoperabilidade entre ambientes informacionais digitais e apontam aspectos imprescindíveis a serem observadas para privilegiar o ranqueamento do registro na SERP, ampliar a visibilidade e possibilitar o reuso dos dados por outros ambientes. Após o aceite, quando da publicação, é importante que todos os metadados do arquivo a ser publicado sejam preenchidos, para que sejam também passíveis de indexação pelos MBAs.

Após a publicação, é possível adotar estratégias e técnicas de ASEO pós-publicação, que Codina (2017) correlaciona ao SEO off-page, visto que o objeto publicado não está mais sob domínio do autor. Contudo, no caso do ASEO, cabe ao autor a adoção de algumas estratégias que auxiliarão na disseminação do conteúdo publicado, promovendo link build e com isso ampliando a visibilidade e as chances de que a publicação seja citada.

Para fins deste estudo, adotamos na Figura 1 a publicação de um objeto acadêmico-científico e não especificamos qual 
a sua tipologia, embora haja a indicação de sistemas que poderiam ser utilizados para a publicação ou disponibilização de ambientes informacionais de publicação de objetos acadêmico-científicos, a exemplo de periódicos e eventos científicos, indexadores de periódicos, repositórios digitais, bibliotecas digitais, portais de dados abertos e dados ligados.

Há, neste contexto a possibilidade de ASEO off-page natural, no caso de periódicos indexados, cujos conteúdos são automaticamente recuperados pelas bases indexadoras, considerando que, há uma fonte primária de publicação e uma base indexadora na qual os objetos são indexados, recuperados pelos metadados e acessíveis pelos links que direcionam para a fonte de publicação.

Torino, Vidotti e Sant'Ana (2019) esclarecem que ao abordar o ciclo de vida de dados elucidam que a fase de recuperação da informação em um ambiente de publicação como um periódico pode alimentar automaticamente a fase de coleta de indexadores de periódicos e repositórios digitais, o que amplia a necessidade de tratamento adequado da representação da informação e da estrutura da camada de dados dos sistemas.

Por outro lado, a Figura 1 elucida que, uma vez publicado o objeto é possível que o autor adote estratégias de ASEO pós-publicação ao utilizar páginas pessoais, redes profissionais ou sociais, currículos on-line, perfil do pesquisador, blogs e demais fontes digitais, utilizando o link de acesso para o conteúdo na fonte original de publicação, o que aumentará o tráfego para a página, a quantidade de páginas externas citando o mesmo conteúdo e com isso o link build.

Codina (2017) considera que após a publicação a disponibilização do objeto em repositório digital permite a ampliação de visibilidade visto que o conteúdo é indexado por estar disponível em um website que possui tráfego. Tal afirmação reforça a viabilidade de publicação em acesso aberto ou da negociação de direito de disponibilização do objeto em repositório digital.

Cabe ainda ao autor a possibilidade de tomar outras ações de disseminação dos conteúdos publicados, tais como: escrever notícias, releases ou resenhas da obra publicada. (CODINA, 2017).

As estratégias e técnicas apresentadas fornecem elementos que poderão ser utilizados pelo MBA para que o crawler colete a produção no momento do rastreamento da web, tornando o conteúdo visível e ranqueado nas primeiras posições na SERP.

Embora este estudo não tenha o objetivo de apresentar a perspectiva do usuário, a Figura 1, indica que, o sujeito interage 
com o MBA por meio de uma interface, na qual representa sua necessidade informacional por meio de uma expressão de busca que aciona o MBA para que a informação indexada pelo crawler seja recuperada e apresentada na SERP e visível para o usuário.

\section{CONSIDERAÇÕES FINAIS}

No contexto acadêmico-científico é notória a crescente valorização pela publicação em fontes de maior impacto e prestígio na área do conhecimento de que trata o conteúdo, fato este que exige esforço por parte do autor, que trabalha na materialização dos resultados da sua pesquisa de forma a apresentá-lo adequadamente à comunidade.

De igual maneira, a velocidade com que conteúdos são publicados na web gera a necessidade da adoção de medidas que assegurem que o conteúdo gerado seja competitivo no momento do ranqueamento pelo $\mathrm{MB}$, neste estudo o MBA, para que esteja dentre os primeiros resultados exibidos na SERP.

Para tanto, é possível que os autores façam uso de estratégias e técnicas de ASEO pré-publicação e pós-publicação para otimizar suas produções visando ao melhor ranqueamento em MBA e com isso alcance maior visibilidade, uso e possibilidade de citação, o que gera a convergência pretendida pela publicação de conteúdos acadêmico-científicos em ambientes web.

Neste sentido, o presente estudo objetivou apresentar estratégias e técnicas ASEO visando apoiar pesquisadores de diferentes áreas para que possam aplicá-las na sua práxis e com isso otimizar seus conteúdos, para que sejam rastreados pelos crawlers, indexados pelos MBAs, apresentados na SERP e citados.

\section{REFERÊNCIAS}

BEEL, J; GIPP, B; WILDE, E. Academic search engine optimization (ASEO): optimizing scholarly literature for Google Scholar and Co. Journal of

Scholarly Publishing, v. 41, n. 2, p. 176-190, 2010. Disponível em: https://docear.org/papers/Academic\%20Search\%20Engine\%20Optimization \%20(ASEO)\%20--\%20preprint.pdf. Acesso em: 07 nov. 2018.

CODINA, L. SEO académico: definición, componentes y guía de herramientas. 2017. Disponível em: https://www.lluiscodina.com/seoacademico-guia. Acesso em: 29 nov. 2018.

ENGE, E.; SPENCER, S.; STRICCHIOLA, J.C. The art of SEO: mastering search engine optimization. California: O'Reilly Media, 2015. 
INGWERSEN, P. Information retrieval interaction. [s.I.]: Taylor Graham London, 1992.

JERKOVIC, J. I. SEO warrior. California: O'Reilly Media, 2010.

LEDFORD, J. L. Search engine optimization bible. Indianapolis: Wiley, 2009.

MONTEIRO, S. D. et al. Sistemas de recuperação da informação e o conceito de relevância nos mecanismos de busca: semântica e significação.

Encontros Bibli, Florianópolis, v. 22, n. 50, p. 161-175, set./dez. 2017.

Disponível em: https://periodicos.ufsc.br/index.php/eb/article/view/15182924.2017v22n50p161/34700. Acesso em: 27 maio 2019.

MONTEIRO, S. D. et al. Em busca da compreensão da "busca" no ciberespaço. In: ENCONTRO NACIONAL DE PESQUISA EM CIÊNCIA DA INFORMAÇÃO, 12., 2011, Brasília.

Anais[...] Brasília: Universidade de Brasília, 2011. p. 2536-2551. Disponível em: http://www.uel.br/grupo-pesquisa/ciberespaco/doc/xii_enancib_busca_2011.pdf. Acesso em: 27 maio 2019.

MOOERS, C. N. Zatocoding applied to mechanical organization of knowledge. Journal of the Association for Information Science and Technology, v. 2, n. 1, p. 20-32, 1951.

OLIVEIRA, H. P. C. de, VIDOTTI, S. A. B. G. Dos ambientes informacionais às ecologias informacionais complexas. Inf. \& Soc.: Est., João Pessoa, v. 26, n. 1, p. 91-101, jan./abr. 2016. Disponível em:

http://www.periodicos.ufpb.br/index.php/ies/article/download/29438/16509. Acesso em: 12 fev. 2019.

TORINO, E.; VIDOTTI, S. A. B. G. Representação da informação e interoperabilidade entre ambientes científicos: um enfoque ao papel do autor. In: SEMINÁRIO EM CIÊNCIA DA INFORMAÇÃO, 8. 2019, Londrina. Anais [...] Londrina: Universidade Estadual de Londrina, 2019. Disponível em: http://www.uel.br/eventos/cinf/index.php/secin2019/secin2019/paper/ viewFile/602/410. Acesso em: 15 out. 2019.

TORINO, Emanuelle; VIDOTTI, Silvana Aparecida Borsetti Gregorio; SANT'ANA, Ricardo César Gonçalves. Ciclo de vida de dados no processo de publicação e acesso à produção científica. In: ENCONTRO NACIONAL DE PESQUISA EM CIÊNCIA DA INFORMAÇÃO, 20. 2019, Florianópolis. Anais [...] Florianópolis: Universidade Federal de Santa Catarina, 2019. Disponível em: https://conferencias.ufsc.br/index.php/enancib/2019/paper/ viewFile/576/612. Acesso em: 15 out. 2019.

Recebido em: 08/01/2019

Aceito em: 08/11/2019 